\title{
The incremental stimulus intensity effect and habituation of evoked electrodermal responses
}

\author{
CHRIS KYRIACOU, DAVID A. T. SIDDLE, JOHN A. SPINKS, \\ DAVID STEPHENSON, and GRAHAM TURPIN \\ University of Southampton, Southampton S09 5NH, England
}

\begin{abstract}
The present paper reports two experiments which were designed to investigate the effectiveness of a gradually increasing series of stimulus intensities for producing response habituation to a higher intensity stimulus. Experiment $1(\mathrm{~N}=45)$ employed 3-sec $1,000-\mathrm{Hz}$ tones of moderate intensity, while Experiment $2(\mathrm{~N}=36)$ employed 1 -sec tones of relatively high intensity. In both experiments, skin conductance was measured while subjects received a series of constant-intensity stimuli (Group C), an incremental series (Group I), or a random series (Group R). Eight trials were presented in Experiment 1, while in Experiment 2, 25 trials (5 blocks of 5 trials) were employed. The range of intensities employed for Groups I and $\mathrm{R}$ was 42-70 dB in Experiment 1, and 80-100 dB in Experiment 2. On the basis of previous work (O'Gorman \& Jamieson, 1975), it was predicted that Group I would display smaller and fewer responses on the final trial in Experiment 1 and during the final trial block in Experiment 2 than would Groups C and R. However, neither experiment provided evidence for the "incremental stimulus intensity effect." In Experiment 1, there were no habituation differences between the groups, while in Experiment 2, the constant series resulted in significantly greater habituation than did the incremental series for both response frequency and response amplitude.
\end{abstract}

Davis and Wagner (1969) reported that a series of stimuli in which intensity was gradually increased resulted in greater habituation than did repeated presentation of the highest intensity stimulus itself. Habituation was measured in terms of the frequency of startle responses in rats to high-intensity white noise. Similar findings were reported by Groves and Thompson (1970) for the hindlimb flexion response in acute spinal cats, and these authors termed the phenomenon the "incremental stimulus intensity effect." According to Groves and Thompson, an incremental stimulus series results in smaller increments in sensitization and hence greater response habituation than does a series of constant-intensity stimuli. Sensitization is said to occur in the "state" system which has been described in terms of the organism's level of arousal. Although Groves and Thompson (1970) have not suggested measures of activity in the state system, their description indicates that commonly used measures of autonomic "arousal" should be appropriate (Graham, 1973).

More recently, O'Gorman and Jamieson (1975) have reported an incremental stimulus intensity effect in human subjects. Exposure to an incremental series of white noise stimuli ranging from 80 to $100 \mathrm{~dB}$ resulted in greater habituation of the skin

This research was supported by Grant B/RG/44047 from the Science Research Council to the second author. Requests for reprints should be addressed to D.A.T. Siddle, PhD, Department of Psychology, University of Southampton, Southampton S09 5NH, United Kingdom. conductance response (SCR) than did exposure to repeated presentations of the $100-\mathrm{dB}$ stimulus itself; habituation was measured in terms of response frequency over the final block of five trials. O'Gorman and Jamieson have argued that their results pose problems for Sokolov's (1963) model of orienting response (OR) habituation. According to Sokolov, the characteristics of previous stimulation are stored in a "neuronal model" with which afferent stimulation is compared. A match between afferent stimulation and the neuronal model leads to OR suppression (habituation). O'Gorman and Jamieson have argued that Sokolov's stimulus matching theory predicts that exposure to the same stimulus will result in the more rapid development of a neuronal model, and hence habituation, than will an incremental series of stimuli. However, two points can be made concerning O'Gorman and Jamieson's study and the conclusions drawn from it. First, Sokolov's model is directed specifically towards explaining the elicitation and habituation of human ORs, and there is evidence that the stimuli employed by O'Gorman and Jamieson elicited defense responses (Graham \& Clifton, 1966). Second, it is not at all clear that O'Gorman and Jamieson have, in fact, demonstrated an incremental stimulus intensity effect with human subjects. The appropriate control condition, which was included in the original Davis and Wagner (1969) experiment, is one in which subjects receive stimuli in the $80-$ to $100-\mathrm{dB}$ range in random order. This condition was not included in the O'Gorman and Jamieson study, and 
thus the difference in responsiveness between the constant intensity and incremental groups could be explained merely in terms of the different stimulus energy to which the subjects were exposed.

The present paper reports two experiments which were designed to investigate these questions.

\section{EXPERIMENT 1}

This experiment was designed to investigate the incremental stimulus intensity effect for stimuli in the OR-eliciting range. The SCR was measured in response to a series of constant-intensity stimuli (Group C), an incremental series (Group I), and a random series (Group R). On the basis of O'Gorman and Jamieson's study, it was predicted that Group I would display smaller and fewer responses on the final trial than would either Group C or Group R. If, on the other hand, the incremental stimulus intensity effect was largely the result of experience with less intense stimuli, it could be predicted that Groups I and $\mathrm{R}$ would both display smaller and fewer responses on the final trial than would Group C, but would not themselves differ significantly.

\section{Method}

Subjects. The subjects were 45 undergraduate volunteers (age range 17-25 years), who were allocated randomly to one of the three groups, with the restriction that each group contain the same proportion of males and females (nine men and six women in each group). Three subjects from Group I and two from each of Groups C and R failed to display an evoked SCR on Trial 1, and were replaced by seven more subjects recruited from the same population.

Apparatus. The stimuli were $1,000-\mathrm{Hz}$ tones of $3 \mathrm{sec}$ duration, presented at a constant interstimulus interval of $40 \mathrm{sec}$ (offset to onset). The tones were produced by a Farnell LFM signal generator and presented via TDH 39 stereophonic earphones; stimulus rise time was $30 \mathrm{msec}$. Stimulus intensity was calibrated (re: .0002 dyne $/ \mathrm{cm}^{2}$ ) using a Brüel and Kjaer artificial ear (Type 4152) and a Brüel and Kjaer microamplifier (Type 2603). Stimulus duration was controlled by a Birkbeck timer.

Bipolar recording of skin conductance was made using domed $\mathrm{Ag}-\mathrm{AgCl}$ electrodes filled with $0.05 \mathrm{M} \mathrm{NaCl}$ electrolyte and attached to masked areas on the index and second fingers of the subject's left hand. The masking collars were placed over the whorls of the fingerprints of the distal phalanges. A constant voltage of $0.5 \mathrm{~V}$ (Lykken \& Venables, 1971) was applied across the electrodes, and conductance was recorded on a Grass Model 7 polygraph with a 7PI B preamplifier sensitivity of $1 \mathrm{~mm}$ of pen deflection equal to $0.02 \mu$ mhos. Respiration was recorded via a Grass nasal thermocouple to detect SCRs arising from respiratory irregularities.

Procedure. The subjects were seated in a semireclining padded chair in a sound-proofed room with an ambient illumination of $2 \mathrm{~lx}$, a temperature range of $19^{\circ}-24^{\circ} \mathrm{C}$, and a relative humidity within the range of $49 \%-56 \%$. The stimulus equipment and recording apparatus were monitored from an adjoining room. The subjects were informed that, after a 5 -min rest period, some tones would be heard from time to time and that no responses were required. They were asked to relax with their eyes closed, to avoid movement as much as possible, but not to go to sleep. After the rest period, the stimulus program commenced without prior warning. Group $\mathrm{C}$ received eight trials at $70 \mathrm{~dB}$. Group I received eight trials beginning at $42 \mathrm{~dB}$ and increasing by $4 \mathrm{~dB}$ each trial to $70 \mathrm{~dB}$ on Trial 8. Group $R$ received the same stimuli as did Group 1. However, the stimuli were randomized for each subject, with the constraints that the stimulus on Trial 6 not be $62 \mathrm{~dB}$ and that the stimuli on Trials 7 and 8 be 66 and $70 \mathrm{~dB}$, respectively. These constraints enabled the intensity increase from Trial 7 to Trial 8 to match that for Group I, without the stimulus on Trial 6 forming an incremental series.

At the end of the testing session, subjects received one presentation of a 3-sec tone of $1,000 \mathrm{~Hz}$ and $100 \mathrm{~dB}$; responses to this stimulus were used for range-correction purposes (Lykken \& Venables, 1971).

Scoring. Any artifact-free SCR greater than or equal to $0.02 \mu$ mhos, occurring from $1-5 \mathrm{sec}$ after stimulus onset, was considered a change evoked by the stimulus. All SCRs elicited on Trial 8 were artifact-free. Where between subject comparisons were made in terms of SCR amplitude, the responses were rangecorrected (Lykken \& Venables, 1971) in terms of each subject's maximum SCR displayed during testing. The maximum SCR occurred to the range-correcting stimulus for 41 of the subjects.

Tonic skin conductance level (SCL) was measured at each minute during the rest period, and mean SCL expressed in terms of $\log (\mu$ mhos $\times 10)$. Tonic SCL was also measured throughout the experimental session at a point $20 \mathrm{sec}$ after each stimulus offset. Spontaneous electrodermal activity was measured during the rest period by counting the number of artifact-free fluctuations greater than or equal to $0.02 \mu$ mhos. Spontaneous activity was also measured throughout the experimental session for each interstimulus interval, excluding the latency period of $1-5 \mathrm{sec}$ after stimulus onset.

\section{Results and Discussion}

There were no group differences in terms of either number of spontaneous fluctuations $[F(2,42)=1.12]$ or tonic SCL $[\mathrm{F}(2,42)<1]$ during the 5-min prestimulus period.

Figure 1 presents the mean range-corrected SCR amplitude for the three groups plotted against trial number. All groups displayed similar negative exponential habituation curves. The Trial 8 amplitude data were analyzed using a one-way analysis of vari-

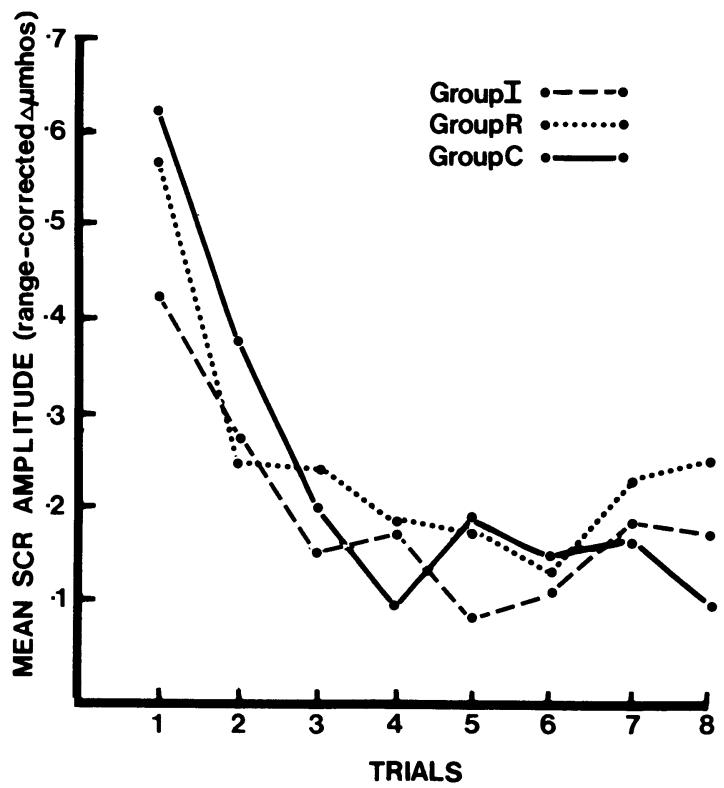

Figure 1. Mean range-corrected SCR amplitude plotted against trials for the three groups. 
ance following a square root transformation. There was no significant difference between the groups $[F(2,42)=2.52]$. Similar results were obtained for the difference in range-corrected SCR amplitude between Trial 1 and Trial $8[\mathrm{~F}(2,42)=2.89]$. Response frequency on Trial 8 was analyzed using chi-square, but no significant effects were obtained $\left[\mathrm{X}^{2}(2)=4.85\right)$. The mean and standard deviation for SCR amplitude, together with the number of SCRs displayed on Trial 8, are shown in Table 1. These results provide no evidence for an incremental stimulus intensity effect for stimuli in the OReliciting range.

Tonic SCL and spontaneous activity throughout the stimulus series were analyzed using two-way analyses of variance in which the main factors were groups and trials. The Geisser-Greenhouse conservative approximation of degrees of freedom was employed for all terms involving repeated measures. For SCL, there were no significant effects for groups $[F(2,42)<1]$, trials $[F(1,42)=1.84]$, or the Groups by Trials interaction $[F(2,42)<1]$. Similar results were obtained for spontaneous activity $[\mathrm{F}(2,42)<1$ for groups, $F(1,42)=1.56$ for trials, and $F(2,42)=$ 1.08 for the interaction]. To the extent that tonic electrodermal activity provides a measure of activity in the state system, these results indicate no group differences in sensitization.

\section{EXPERIMENT 2}

This experiment was designed to investigate the incremental stimulus intensity effect for highintensity stimuli. The SCR was measured in response to a series of constant-intensity stimuli (Group C), an incremental series (Group I), and a random series (Group R). The addition of Group R allows the effects of an incremental series and exposure to lower intensity stimuli to be assessed separately. On the basis of O'Gorman and Jamieson's (1975) study, it was predicted that Group I would display smaller and fewer responses during the final trial block than would either Group C or Group R. If, on the other

Table 1

Number of Responses and the Mean and Standard Deviation for SCR Amplitude on the Last Trial in Experiment 1, Together with Means and Standard Deviations for Frequency and Amplitude of SCRs During the Last Trial Block in Experiment 2

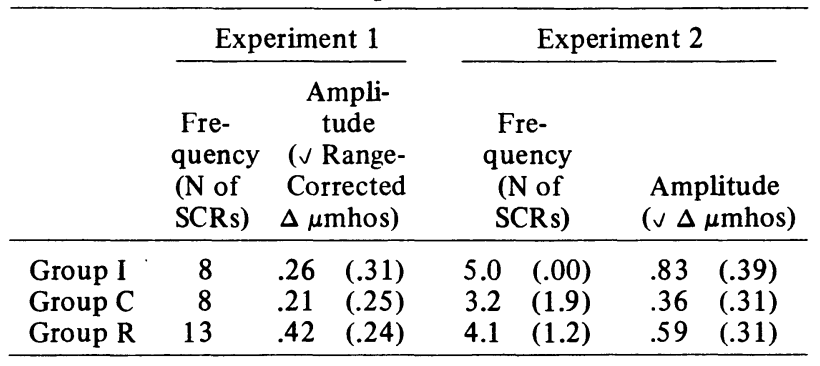

hand, the incremental stimulus intensity effect was largely the result of experience with lower intensity stimuli, it could be predicted that Groups I and R would both display smailer and fewer responses during the final trial block than would Group C, but would not themselves differ significantly.

\section{Method}

Subjects. The subjects were 36 undergraduate volunteers (age range 17-26 years), who were allocated randomly to one of the three groups, with the restriction that each group contain the same proportion of males and females (seven men and five women in each group). One subject from Group $\mathrm{R}$ failed to display an evoked SCR on Trial 1 and was replaced by another subject recruited from the same population.

Apparatus. The stimuli were $1,000-\mathrm{Hz}$ tones of $1 \mathrm{sec}$ duration, presented at a constant interstimulus interval of $40 \mathrm{sec}$ (offset to onset); stimulus rise time was $30 \mathrm{msec}$. Control of stimulus presentation, the recording techniques, and the recording apparatus were the same as in Experiment 1.

Procedure. The procedure was essentially the same as in Experiment 1, except that different intensity schedules were employed. Group C received 25 trials at $100 \mathrm{~dB}$. Group I received five blocks of five stimuli; the first block consisted of five tones at $80 \mathrm{~dB}$, and the intensity was increased by $5 \mathrm{~dB}$ for each successive trial block such that the final trial block consisted of five tones at $100 \mathrm{~dB}$. Group $\mathrm{R}$ received 20 trials consisting of five trials at each of $80,85,90$, and $95 \mathrm{~dB}$. These stimuli were randomized for each subject, with the constraint that each trial block of five tones contain at least one of each of the four intensity levels. This resulted in one of the intensity levels occurring twice within each trial block. These 20 trials were then followed by 5 trials at $100 \mathrm{~dB}$.

All aspects of electrodermal activity were scored in the same manner as in Experiment 1, except that no range-correction procedures were adopted.

\section{Results}

There were no group differences in terms of either number of spontaneous fluctuations $[F(2,33)<1]$ or tonic $\operatorname{SCL}[\mathrm{F}(2,33)=1.14]$ during the $5 \mathrm{~min}$ prestimulus period.

The mean frequency and amplitude of responaing during the last trial block are shown in Table 1. A Kruskal-Wallis analysis of variance of the frequency data indicated significant group differences $[\mathrm{H}(2)=$ $13.30, \mathrm{p}<.01$ ], and it can be seen that Group $\mathrm{C}$ displayed the lowest number of SCRs, followed by Groups $R$ and I. In fact, Group I displayed no habituation.

Figure 2 presents mean SCR amplitude for the three groups plotted against trial blocks. All groups displayed a similar decrement in responding during the first three trial blocks, but whereas Groups $\mathrm{C}$ and $R$ continued to display a decrement on Block 4, Group I responded more vigorously. On Block 5, Group I displayed even greater responding, and there was also an increase in Group R's responsiveness. The data from Trial Block 5 were analyzed using a one-way analysis of variance following a square root transformation. There was a reliable effect for groups $[F(2,33)=5.78, p<.01]$, and further analysis (Newman-Keuls test) indicated that 


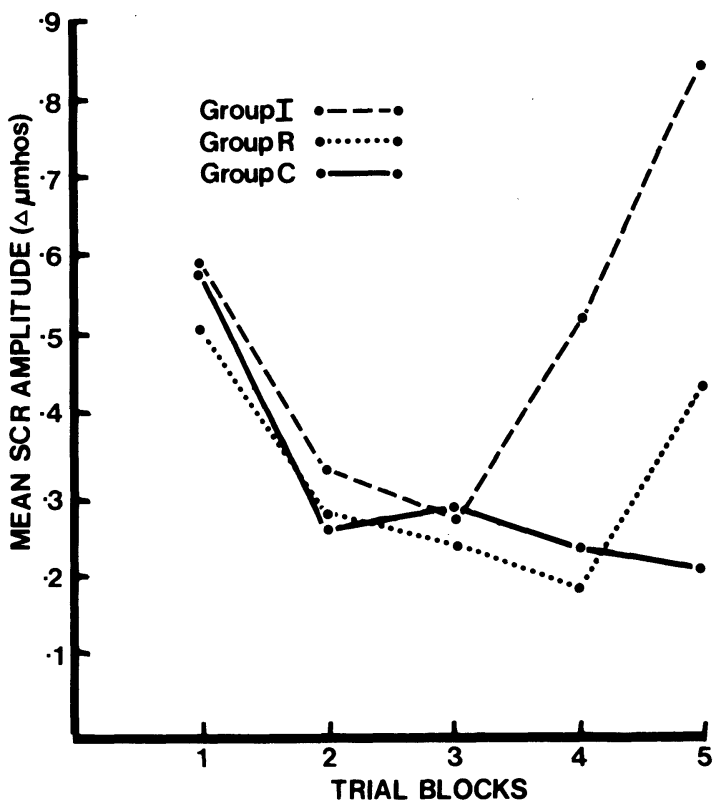

Figure 2. Mean SCR amplitude plotted against trial blocks for the three groups.

Group I displayed significantly larger SCRs than did Group C $(p<.01)$. No other comparisons were significant.

The difference in mean response amplitude between Trial Block 1 and Trial Block 5 was obtained for each subject. Positive scores (indicating habituation) were obtained for all subjects in Group C and all but five subjects in Group R, while negative scores (indicating increased responsiveness) were obtained for all but one subject in Group I. A one-way analysis of variance revealed a reliable effect for groups $[F(2,33)=9.49, \mathrm{p}<.01]$. Further analysis (Newman-Keuls test) indicated that Group I displayed significantly greater responsiveness than did Groups $C(p<.01)$ and $R(p<.05)$, and that Group $\mathbf{R}$ displayed significantly greater responsiveness than did Group $C(p<.05)$.

Tonic SCL and spontaneous activity throughout the stimulus series were analyzed using two-way analyses of variance in which the main factors were groups and trials. The spontaneous activity data were subjected to a square-root transformation prior to analysis. For SCL, there were no significant effects for groups $[F(2,33)=1.06]$, trials $[F(1,33)=2.75]$, or the Groups by Trials interaction $[\mathrm{F}(2,33)=2.82]$. For spontaneous activity, there was a significant effect for trials $[F(1,33)=8.94, p<.01]$, but no significant effects for groups $[F(2,33)<1]$ or the Groups by Trials interaction $[\mathrm{F}(2,33)<1]$. To the extent that tonic electrodermal activity provides a measure of activity in the state system, these results indicate no group differences in sensitization throughout the stimulus series.

\section{GENERAL DISCUSSION}

The present results provide no evidence for an incremental stimulus-intensity effect. This finding held for moderate intensity stimuli within the OReliciting range and also for higher intensity stimuli which might be expected to elicit DRs. In Experiment 1 , there were no differences in habituation between the groups which had received a constant, incremental, or random series, while in Experiment 2, the constant series resulted in greater habituation than did the incremental. These findings held for both response frequency and response amplitude.

O'Gorman and Jamieson (1975) have argued that the incremental stimulus intensity effect poses problems for Sokolov's (1963) model of OR habituation. However, the results of Experiment 1 indicate that the incremental stimulus intensity effect does not occur for stimuli within the OR-eliciting range. Nevertheless, stimuli within the OR range evoke responses which are subject to rapid habituation, and the fact that a number of subjects did not display measurable responses towards the end of the stimulus series may have obscured any experimental effects.

It is the interpretation of the results of Experiment 2 which poses greater problems concerning the incremental stimulus intensity effect in human subjects. Contrary to O'Gorman and Jamieson's (1975) results, the constant group in the present study displayed greater habituation than did the incremental group. However, the present study employed pure tones rather than white noise, and it could be argued that as white noise is more aversive than tones (Graham \& Slaby, 1973), the stimuli employed by O'Gorman and Jamieson were more resistant to habituation. If this was the case, it might be expected that the incremental group in the present study would display greater habituation than did the incremental group in their study. In fact, inspection of Figure 1 in O'Gorman and Jamieson's report (p. 387) indicates that the mean frequency of responding during Trial Block 5 was substantially lower than that reported here. Another difference between the studies concerns the type of subjects employed. In O'Gorman and Jamieson's experiment, the subjects were graduates and technicians, while the present study employed naive undergraduates. It could be argued that the naive subjects in Group I were made more anxious than those in Group $\mathrm{C}$ by not knowing how intense the tones would become. However, to the extent that an increase in anxiety should be reflected by an increase in tonic electrodermal activity (Gatchel \& Gaas, 1976), this explanation is not supported by the data.

It is not clear how the results of Experiment 2 would be interpreted in terms of Groves and Thompson's (1970) dual-process theory of habituation. These authors have proposed that response 
habituation is the net outcome of two inferred processes-habituation, which is said to occur in the S-R pathway, and sensitization, which is said to occur in the state system. According to Groves and Thompson, an incremental series results in smaller increments in sensitization than does a series of constant intensity stimuli, and hence greater response habituation. To the extent that tonic electrodermal activity provides a measure of activity in the state system, this study reports no group differences in sensitization. If the greater response habituation following a constant series in Experiment 2 is not the result of differences in sensitization, then it must be argued that it is the result of greater habituation in the S-R pathway for the constant group. If this was the case, a constant series should also have resulted in greater response habituation in the O'Gorman and Jamieson study, since they also reported no differences in sensitization. This did not occur, and it is thus difficult to see how the dualprocess theory can account for both sets of data.

Examination of SCR amplitude throughout Experiment 2 indicates that the difference between Groups $\mathrm{C}$ and I on Trial Block 5 was the result of more vigorous responding by Group I. Figure 2 clearly shows that although there was very little difference between Groups C and I on Trial Block 3, Group I responded more strongly during Trial Block 4, and even more vigorously during Trial Block 5, while Group C displayed more habituation. One explanation of these data might be that the stimuli presented on Trial Blocks $4(95 \mathrm{~dB})$ and 5 (100 dB) evoked more DRs for Group I than did the stimuli presented on Trial Block 3 ( $90 \mathrm{~dB})$.

A group which received stimuli in a random order was employed in each experiment in order to investigate whether any difference between the constant and incremental series could be accounted for in terms of experience with tones of lower intensities.
In Experiment 1, Group R did not differ significantly from Groups $C$ and I. In Experiment 2, however, Group R showed significantly more habituation than did Group I but significantly less habituation than did Group C, when habituation. was measured in terms of the difference in the mean response amplitude displayed on Trial Blocks 1 and 5. No explanation is readily available to account for the fact that Group $\mathbf{R}$ displayed greater habituation than did Group I.

\section{REFERENCES}

Davis, M., \& W AGNER, A. R. Habituation of startle response under incremental sequence of stimulus intensities. Journal of Comparative and Physiological Psychology, 1969, 67, 486-492.

GATCHEL, R. J., \& GAAS, E. Effects of arousal level on short- and long-term habituation of the orienting response. Physiological Psychology, 1976, 4, 66-68.

GraHAM, F. K. Habituation and dishabituation of responses innervated by the autonomic nervous system. In H. V. S. Peeke \& M. J. Herz (Eds.), Habituation (Vol. 1) Behavioral studies. New York: Academic Press, 1973. Pp. 163-218.

Graham, F. K., \& Clifton, R. K. Heart-rate change as a component of the orienting response. Psychological Bulletin, 1966, 65, 305-320.

Graham, F. K., \& Slaby, D. A. Differential heart rate changes to equally intense white noise and tone. Psychophysiology, 1973, 10. 347-362.

Groves, P. M., \& Thompson, R. F. Habituation: A dual process theory. Psychological Review', 1970, 77, 419-450.

Lykken, D. T., \& Venables, P. H. Direct measurement of skin conductance: A proposal for standardization. Psychophysiology, 1971, 8. 656-672.

O'Gorman, J. G., \& Jamieson, R. D. The incremental stimulus intensity effect and habituation of autonomic responses in man. Physiological Psychology, 1975, 3, 385-389.

Sokolov, E. N. Perception and the conditioned reflex. Oxford: Pergamon Press, 1963.

(Received for publication September 21, 1976; accepted October 15, 1976.) 